\title{
Modelo explicativo de la autoeficacia académica: autorregulación de actividades, afecto positivo y personalidad
}

\section{Explanatory model of academic self-efficacy: self-regulation of activities, positive affection, and personality}

Daniel Edgardo Yupanqui-Lorenzo*

Universidad César Vallejo, Perú

ORCID: https://orcid.org/0000-0002-8977-2888

Fiorella Mariel Mollinedo Flores

Universidad Privada del Norte, Perú

ORCID: https://orcid.org/0000-0002-9307-592X

Aissa Celeste Montealegre Echaiz

Universidad Inca Garcilaso de la Vega, Perú

ORCID: https://orcid.org/0000-0002-0256-7516

Recibido11-03-20 Revisado 23-04-21 Aceptado 28-06-21 En línea 06-09-21

*Correspondencia

Email: dyupanquil@ucvvirtual.edu.pe
Citar como:

Yupanqui-Lorenzo, D. E., Mollinedo, F. M., \& Montealegre, A. C. (2021). Modelo explicativo de la autoeficacia académica: autorregulación de actividades, afecto positivo y personalidad. Propósitos y Representaciones, http://dx.doi.org/10.20511/pyr2021.v9n2.755 


\section{Resumen}

La autoeficacia académica es un potente predictor del éxito académico, sin embargo, existen diversos factores que lo predisponen. Debido a ello se propone un modelo explicativo basado en la autorregulación de actividades académicas, afecto positivo y personalidad. Se utilizó regresión lineal múltiple jerárquico para sustentar la propuesta predictiva del modelo. Se utilizaron la Escala de Autoeficacia Percibida Específica de Situaciones Académicas, The Scale of Positive and Negative Experience, Subescala Autorregulación de actividades de la Escala de Procrastinación Académica, y Mini International Personality Item Pool (MINI-IPIP). Se obtuvieron altas correlaciones en la autorregulación de actividades, afecto positivo y personalidad (extraversión, responsabilidad y apertura) para con la autoeficacia académica. El modelo predictivo final, explicó el $30.1 \%$ de la varianza total de la autoeficacia académica. En conclusión, el modelo propuesto mediante revisión de la literatura es un fuerte predictor de la autoeficacia académica, y promueve el desarrollo de creencias auto eficaces.

Palabras clave: Autoeficacia, Big Five, tipos de personalidad, afecto positivo, autorregulación académica.

\section{Summary}

Academic self-efficacy is a powerful predictor of academic success, yet there are several factors that predispose it. Because of this, an explanatory model is proposed using the self-regulation of academic activities, positive affection, and personality. Hierarchical multiple linear regression was used to support the predictive proposal of the model. The Specific Perceived Self-Efficacy Scale of Academic Situations, The Scale of Positive and Negative Experience, Subscale SelfRegulation of Activities of the Academic Procrastination Scale, and Mini International Personality Item Pool (MINI-IPIP) were used. High correlations were obtained in the selfregulation of activities, positive affection, and personality (extraversion, concientousness and openness to experience) to academic self-efficacy. The final predictive model explained $30.1 \%$ of the total variance of academic self-efficacy. In conclusion, the model proposed through literature review is a strong predictor of academic self-efficacy and promotes the development of self-effective beliefs.

Keywords: Self-efficacy, Big Five, personality traits, positive affect, academic self-regulation.

\section{Introducción}

La realidad educativa en el campo universitario ha mostrado que los estudiantes inician sus estudios en la etapa de la adolescencia, sin haber logrado competencias académicas adecuadas (Pease, 2015; Pecina, 2014). Es en la universidad donde el estudiante necesita mejorar sus capacidades cognitivas (memoria, percepción, atención, concentración, etc.) para contribuir en su aprendizaje (Moraine, 2014). Sin embargo, existen factores personales como la autoeficacia, motivación, emoción y personalidad que influyen en las aspiraciones educativas y el desempeño académico (Caprara, Barbaranelli, Pastorelli, \& Cervone, 2004; Rottinghaus, Lindley, Green, \& Borgen, 2002). Desde dicha perspectiva, el estudio se estructura hacia un modelo integrador sobre la autoeficacia académica.

\section{Autoeficacia en el contexto académico}

En principio, la autoeficacia es concebida como un conjunto de creencias personales sobre las propias capacidades (Bandura, 1997); lo que genera autoconfianza, aptitudes y actitudes de logro (Zimmerman \& Kitsantas, 2005). Esto permite que las personas ejecuten procesos cognitivos, conductuales y sociales para encontrar estrategias y perseverar en la solución de conflictos (Bandura, 1986). El estudio sobre efecto de la autoeficacia, como mediador y predictor, ha 
permitido inquirir dentro de los contextos clínicos (Sheu, Liu, \& Li, 2017) y educacionales (Cupani \& Zalazar-Jaime, 2014; Narváez, Posso, Guzmán, \& Valencia, 2018).

Palenzuela (1983) introdujo la autoeficacia en el contexto educativo, y desde entonces se han mostrado aportes psicométricos (Dominguez-Lara et al., 2012; Dominguez, 2014; Ornelas, Blanco, Rodríguez, \& Flores, 2011) y aplicativos (Kurtovic, Vrdoljak, \& Idzanovic, 2019; Lee \& Ciftci, 2014; Ornelas, Blanco, Gastélum, \& Chávez, 2012; Ümmet, 2017; Zhang, 2016). Además, se ha demostrado que la autoeficacia repercute en la motivación y en las estrategias académicas (Bandura, 2006; Ruiz-Dodobara, 2005; Schunk, 1991). Incluso, tiene el poder de estimular el aprendizaje y, por consiguiente, el éxito académico (Galindo, 2004; Valdivieso, Carbonero, \& Martín-Antón, 2013); evidenciándose un proceso cíclico, es decir, el estudiante obtiene éxito por ser auto eficaz, y el éxito es un refuerzo positivo a su autoeficacia (Bonetto, Paoloni, \& Donolo, 2017).

Diversos estudios concluyen que los estudiantes que mantienen alta autoeficacia tienen confianza en sus habilidades para lograr objetivos de aprendizaje (Zuffianò et al., 2013) y tener éxito en su vida académica (Criollo, Romero, \& Fontaines-Ruiz, 2017). Igualmente, la autoeficacia, junto con la autoestima, han llegado a ser predictores de la capacidad creativa del estudiante (Zarza-Alzugaray, Bustamante, Casanova, \& Orejudo, 2018).

De esta manera, la autoeficacia promueve conductas regulativas para demostrar su mejor desempeño, tal como la planificación y organización (Zimmerman, Kitsantas, \& Campillo, 2005). Ciertos factores como la autorregulación de actividades académicas mantienen relación estrecha con la autoeficacia cognitiva (creencias) y conductual (actitudes y comportamientos) (Alegre, 2014; Brígido \& Borrachero, 2011; Fernández \& Bernardo, 2011). Conjuntamente, la autoeficacia y autorregulación del aprendizaje son predictoras del $43 \%$ de varianza de las metas estudiantiles (Covarrubias-Apablaza, Acosta-Antognoni, \& Mendoza-Lira, 2019). Por lo tanto, la autorregulación de actividades académicas se considera de naturaleza conductual, y la literatura sustenta su relación con la autoeficacia. En base a estos aportes, se planteó la primera hipótesis de investigación; en la que se resalta la autorregulación de actividades como factor predictor de la autoeficacia.

H1. El modelo 1, con la autorregulación de actividades, predice la autoeficacia académica.

\section{Relación de la autoeficacia y el afecto}

La autoeficacia por su naturaleza teórica se trata de un conjunto de creencias, las cuales están influenciados por el estado anímico. Por ello, algunas variables de naturaleza cognitivoemocional como la autoestima se han relacionado moderadamente con la autoeficacia (Reina, 2017; Zarza-Alzugaray et al., 2018). La autoestima se compone por autopercepciones que están influenciadas por el estado emocional; por lo tanto, el juicio de la persona con respecto a sus virtudes físicas e, inclusive, habilidades personales se encuentran afectadas por su estado afectivo (Fodor \& Pintea, 2017). Es así como la autoeficacia posee una estrecha relación con variables de naturaleza emocionales.

Para este estudio se considera al afecto como una respuesta a un estímulo (Rottenberg \& Johnson, 2007), es decir, se trata de una emoción momentánea (Rottinghaus, Jenkins, \& Jantzer, 2009), el cual se encuentra conectado con la atención, memoria y el procesamiento de información (Bower, 1981; Eich, 1995). Es decir, el estado emocional positivo de un estudiante puede beneficiar el proceso de aprendizaje y éxito académico (Bandura, 1997); en cambio, si tiene emociones negativas, se producen problemas en la atención, concentración y memoria, elementos necesarios para el aprendizaje. Otros estudios han mostrado que el afecto positivo también favorece la salud física, psicológica y social de las personas (Krok \& Zarzycka, 2020). En contraparte, el afecto negativo genera malestar en las diferentes áreas de vida (He, Zhou, Zhao, 
Jiang, \& Wu, 2020), por lo que su importancia en el presente estudio es trascendente para comprender la autoeficacia.

La influencia del afecto positivo en la autoeficacia causa conductas y comportamientos que benefician el desempeño del estudiante que busca la autorrealización personal (Shepherd \& Patzelt, 2018). Además, ha servido como un mediador de la autoeficacia y la conducta (He et al., 2020). Por otra parte, se ha demostrado que mientras el afecto positivo aumenta también lo hace la autoeficacia y satisfacción (Schutte, 2013). Inclusive, el afecto positivo se relacionó con la autoeficacia social, autoestima, optimismo, satisfacción por la vida y felicidad; además de ser un predictor robusto de los mismos (Caprara, Steca, Gerbino, Paciello, \& Vecchio, 2006). A razón de lo planteado y a falta de literatura que muestre el poder predictivo del afecto positivo en la autoeficacia, se establece la hipótesis dos que integra al afecto positivo dentro del modelo explicativo del estudio. Siendo el afecto positivo el componente emocional del modelo.

H2. El modelo 2, con la autorregulación de actividades y afecto positivo, predice la autoeficacia académica.

\section{Aportes de la personalidad y autoeficacia}

En cuanto a la personalidad se ha obtenido correlaciones estadísticamente significativas con factores educativos (Alvarado-Ochoa, González-Gordillo, Fontaines-Ruiz, Salamea-Nieto, \& Blacio-Aguilar, 2018; Poropat, 2009), así mismo, se ha relacionado con la autoeficacia (Lin, Lu, Chen, \& Chen, 2014; Özdemir et al., 2019; Rottinghaus et al., 2002). Estudios posteriores han mostrado que la autoeficacia se relacionaba de manera positiva con la extraversión, responsabilidad y apertura como factores de mayor relevancia de la personalidad (Zakiei, Vafapoor, Alikhani, Farnia, \& Radmehr, 2020); sin embargo, la amabilidad fue el factor de menor relación con la autoeficacia (Judge \& Ilies, 2002), lo que permite concluir que la amabilidad no es un factor determinante, de modo que, es el primer tipo de la personalidad que se excluye en el análisis. El neuroticismo mantuvo correlaciones significativas pero negativas con la autoeficacia (Zakiei et al., 2020), por lo que también se excluye por su efecto negativo dentro de la autoeficacia académica.

La personalidad y la autoeficacia son predictores del desempeño académico (Cupani \& Zalazar-Jaime, 2014) y otras variables académicas (Nawas, Khattak, \& Rehman, 2019; Sidratulmunthah, Hussain, \& Imran-Malik, 2018). Estudios han mostrado que la autoeficacia es un mediador de la personalidad con factores académicos (Caprara, Vecchione, Alessandri, Gerbino, \& Barbaranelli, 2011; Fosse, Buch, Säfvenbom, \& Martinussen, 2016). Cabe señalar que las personas con personalidad proactiva muestran altos índices de autoeficacia (Francescato, Lauriola, Giacomantonio, \& Mebane, 2020), de modo que la relación entre ambas variables queda determinada (Zakiei et al., 2020). Producto de ello, se planteó la hipótesis tres que integra la personalidad (extraversión, responsabilidad y apertura) en el modelo predictivo de la autoeficacia.

H3. El modelo 3, con la autorregulación de actividades, afecto positivo y personalidad (extraversión, responsabilidad y apertura), predice la autoeficacia académica.

\section{Método}

\section{Participantes}

La selección de los participantes fue a través de un muestreo probabilístico aleatorio simple (Kerlinger \& Lee, 2002) de una población total 2500 estudiantes. Se utilizó un nivel de confianza del $99 \%$ y un margen de error del 2\% para una mayor representatividad de las inferencias en los resultados. Se obtuvo una muestra de 1562 estudiantes universitarios de la ciudad de Lima. Los estudiantes pertenecieron a diferentes carreras en el área de salud, ingeniería, ciencias sociales y humanidades. Luego de aplicar los instrumentos se eliminaron 338 encuestas por no cumplir con 
los estándares de calidad requeridos. Finalmente se obtuvo una muestra final de 1224 estudiantes evaluados.

Las características sociodemográficas de la muestra revelaron que el $61.5 \%$ fueron mujeres y $38.5 \%$, hombres. Según la edad se compone un $52.1 \%$ por jóvenes, $40 \%$ adolescentes, $6.9 \%$ jóvenes adultos y $1.1 \%$ por adultos $(\mathrm{M}=22.89$ años; $\mathrm{DE}=5.47$ años $)$. En su mayoría fueron solteros y en relación (75.6\% y $20.5 \%$, respectivamente). Gran parte de la muestra solo se dedica a sus estudios $(52.4 \%)$, mientras otros trabajan medio tiempo (11.4\%), tiempo completo (26.6\%) $\mathrm{y}$ de forma independiente $(0.4 \%)$.

\section{Tabla 1.}

Características demográficas de los participantes

\begin{tabular}{llcc}
\hline \multicolumn{1}{c}{ Categoría } & \multicolumn{1}{c}{ Grupos } & $f$ & $\%$ \\
\hline Sexo & Hombre & 471 & $38.5 \%$ \\
& Mujer & 753 & $61.5 \%$ \\
Edad & $15-20$ años & 489 & $40.0 \%$ \\
& $21-30$ años & 638 & $52.1 \%$ \\
& $31-40$ años & 84 & $6.9 \%$ \\
& $41-49$ años & 13 & $1.1 \%$ \\
Estado civil & Soltero & 925 & $75.6 \%$ \\
& En relación & 251 & $20.5 \%$ \\
& Casado & 43 & $3.5 \%$ \\
& Divorciado & 5 & $0.4 \%$ \\
Laboral & Solo estudia & 641 & $52.4 \%$ \\
& Medio tiempo & 140 & $11.4 \%$ \\
& Tiempo completo & 325 & $26.6 \%$ \\
& Independiente & 118 & $9.6 \%$ \\
\hline
\end{tabular}

Nota: $f=$ frecuencia; $\%=$ porcentaje.

\section{Instrumentos}

\section{Escala de Autoeficacia Percibida Específica de Situaciones Académicas (EAPESA)}

La escala fue elaborada por Palenzuela (1983), quien publicó su versión de 10 ítems. Cada ítem se responde en una escala tipo Likert con cinco opciones de respuesta (1=Totalmente en desacuerdo a $5=$ Totalmente de acuerdo). Dicha escala pasó por una adaptación lingüística, luego se procedió con un AFE, el cual agrupó los 10 ítems en un factor general (Dominguez-Lara et al., 2012). Así mismo, se realizó un AFC que indicó que había adecuado índices de ajuste (RMSEA= $.056 ; \mathrm{RMR}=.029 ; \mathrm{GFI}=.969, \mathrm{CFI}=.978 ; \mathrm{AGFI}=.949)$ y confiabilidad $(\alpha=.88)$ en la unidimensionalidad de la escala (Dominguez, 2014).

\section{The Scale of Positive and Negative Experience (SPANE)}

SPANE mide el afecto positivo y negativo de las personas. Está compuesto por 12 ítems, 6 de ellos responden al afecto positivo (AP) y los otros 6 pertenecen al afecto negativo (AN). La escala de respuesta es de cinco puntos ( $1=$ muy raramente o nunca, a $5=$ muy a menudo o siempre) 
(Diener et al., 2010). Se realizó traducción al español y se utilizó el AFE en una muestra peruana (p<.05), mostrando que la AP explicaba el 69,5\% de la varianza y el AN el 61.5\% de la varianza; y la confiabilidad del AP fue .91 y del AN de .87 (Cassaretto \& Martínez-Uribe, 2017).

\section{Subescala Autorregulación de actividades de la Escala de Procrastinación Académica}

La Escala de Procrastinación Académica se compone por dos factores: Autorregulación de actividades ( 9 ítems) y Postergación de actividades (3 ítems) (Dominguez-Lara, Villegas, \& Centeno, 2014). Se realizó AFE y AFC para el instrumento. El cual obtuvo adecuados índices de ajuste en el modelo bifactor (RMSEA= .078; RMR= .064; GFI= .97; AGFI= .96), mostrando independencia de ambos factores. Se eligió la subescala: Autorregulación de actividades (AA), por su composición de ítems positivos que reflejan planeamiento, responsabilidad, puntualidad, gestión de tiempo y automotivación en situaciones académicas; y también por la cantidad de ítems que fueron adecuados para la aplicación en la muestra objetivo.

\section{Mini International Personality Item Pool - Five Factor Model Measure (MINI-IPIP)}

Esta escala fue elaborada por Donnellan et al. (2006), de la versión Big Five IPIP de Goldberg (1992), las cuales muestran cinco tipos de personalidad: Responsabilidad (R), Apertura (A), Extraversión (E), Amabilidad (Ab) y Neuroticismo (N). Se compone por 20 ítems los cuales se distribuyen equitativamente para cada factor. La escala de respuesta varía del $1=$ Totalmente en desacuerdo a 5=Totalmente de acuerdo. Su traducción al español fue descrita por MartínezMolina \& Arias (2018), quienes realizaron su propia adaptación de los ítems inversos a ítems positivos. Se mostró adecuado ajuste en el AFC (RMSEA = .052; CFI= .984; TLI= .970; $\left.x^{2}=171\right)$. Sin embargo, para el presente estudio se realizó un AFC con una muestra de estudiantes universitarios peruanos, obteniendo adecuados índices de ajuste (RMSEA= .03; SRMR= 03 ; $\mathrm{CFI}=.97 ; \mathrm{TLI}=.96)$ y adecuado coeficiente de confiabilidad para cada factor: $\mathrm{R}(\omega=.80), \mathrm{A}$ $(\omega=.79), \mathrm{E}(\omega=.71), \mathrm{Ab}(\omega=.80)$ y $\mathrm{N}(\omega=.51)$.

\section{Procesamiento}

Debido al contexto de pandemia, la aplicación de los instrumentos fue de forma virtual. Para ello, se virtualizaron los instrumentos en Google Formulario. En dicho formulario se expuso las motivaciones de la investigación, el propósito del estudio, la finalidad y justificación del estudio. Conjuntamente se brindó un consentimiento informado donde el estudiante debía marcar la aceptación voluntaria para participar en la investigación. Para aquellos que decidieron participar, se aseveró que los datos de los participantes serán de uso exclusivo de la investigación; asimismo, se aseguró el anonimato de su participación. La distribución de las encuestas estuvo a cargo de los investigadores del estudio, quienes tuvieron un intervalo de tiempo de tres semanas para recolectar la información de los estudiantes.

\section{Análisis de datos}

Se examinó la data para evitar datos perdidos, duplicados o respuestas nulas. Se utilizó R Studio para el procesamiento y cálculo de los resultados. Se utilizó los valores de asimetría y curtosis \pm 1 como representación de la normalidad (George \& Mallery, 2020; Kline, 2016). Por consiguiente, se procedió al cálculo de estadísticos descriptivos e inferenciales (correlación y regresión lineal múltiple) para responder a las hipótesis. Para la interpretación de los coeficientes de correlación se utilizó la categorización de Cohen (1988): <.10 (débil), . 30 (moderado) y >.50 (fuerte). Se aplicó un análisis de regresión jerárquico, utilizando los modelos planteados en las hipótesis. Para ello se calcularon el coeficiente B y beta estandarizado para conocer la importancia relativa de las variables en cada modelo respecto a la variable dependiente. Además, se obtuvieron el coeficiente de correlación y determinación el cual brinda información sobre la varianza explicada del efecto de las variables independiente en la dependiente (George \& Mallery, 2020). Por último, se calculó el tamaño del efecto por el $f^{2}$ de Cohen (1988) para regresiones múltiples, con los siguientes puntos de corte: $0.02-0.15$ (débil), $0.15-.35$ (moderado) y >.35 (fuerte). 


\section{Resultados}

Considerando el análisis de la normalidad se evaluó los histogramas de residuos, la homocedasticidad de los datos; además, los datos de asimetría y curtosis se encontraron dentro del umbral aceptable \pm 1 (George \& Mallery, 2020). Por lo tanto, la tabla 2 presenta la media, desviación estándar y las correlaciones sobre las variables de interés. Como se esperaba la autoeficacia académica correlacionó moderadamente con la autorregulación de actividades $(\mathrm{r}=.43)$, afecto positivo $(\mathrm{r}=.41)$, responsabilidad $(\mathrm{r}=.31)$, apertura $(\mathrm{r}=.30)$, y con la única variable que tuvo una relación pequeña fue con extraversión ( $\mathrm{r}=.23$ ). Además, se estudiaron los modelos planteados y se comprobó que cumplen el supuesto de independencia (Durbin-Watson $=2.05$ ) y no colinealidad (tolerancia $=.883, \mathrm{VIF}=1.13$ ) lo que permitió realizar el análisis de regresión.

\section{Tabla 2}

Media, desviación estándar y correlaciones

\begin{tabular}{lrrrrrrr}
\hline \multicolumn{1}{c}{ Variable } & $M$ & $S D$ & 1 & 2 & 3 & 4 & 5 \\
\hline 1. Autoeficacia académica & 38.98 & 6.38 & & & & & \\
2. Autorregulación de actividades & 34.15 & 5.57 & $.43^{* *}$ & & & & \\
3. Afecto positivo & 23.24 & 4.36 & $.41^{* *}$ & $.37^{* *}$ & & & \\
4. Extraversión & 12.07 & 3.22 & $.23^{* *}$ & $.08^{* *}$ & $.29^{* *}$ & & \\
5. Responsabilidad & 14.35 & 3.16 & $.31^{* *}$ & $.53^{* *}$ & $.32^{* *}$ & $.07^{*}$ & \\
6. Apertura & 14.46 & 2.82 & $.30^{* *}$ & $.21^{* *}$ & $.24^{* *}$ & $.26^{* *}$ & $.18^{* *}$ \\
\hline Nota: ${ }^{*}$ indica $p<.05 ;{ }^{* *}$ indica $p<.01$. & & & & & & &
\end{tabular}

Se puso a prueba tres modelos que se fueron armando según las hipótesis planteadas en el estudio. La tabla 3 muestra los resultados del modelo 1, el cual se compone por la autorregulación de actividades como variable independiente y la autoeficacia académica como dependiente. Estos resultados mostraron el efecto predictivo de la autorregulación de actividades $(\beta=.43, \mathrm{t}=16.84, \mathrm{p}<.000)$ en la autoeficacia académica, explicando el $19 \%$ de su varianza. El tamaño del efecto del modelo con un solo predictor fue moderado $\left(f^{2}=.24\right)$.

El modelo 2 añade al primer modelo el afecto positivo como variable independiente. Los resultados de este modelo muestran el poder predictivo de ambas variables: autorregulación de actividades $(\beta=.32, \mathrm{t}=12.26, \mathrm{p}<.000)$ y afecto positivo $(\beta=.29, \mathrm{t}=11.04, \mathrm{p}<.000)$, los cuales explican el $26.2 \%$ de la varianza en la autoeficacia académica, incrementando un $7.4 \%$ del modelo anterior. El tamaño del efecto para este modelo incrementó su valor a comparación del modelo anterior, siendo un tamaño del efecto fuerte $\left(f^{2}=.36\right)$

Por último, inicialmente se calculó el modelo 3, donde se incluyó los tres tipos de personalidad al modelo anterior. La responsabilidad, extraversión y la apertura como tipos de personalidad muestran su aporte al modelo predictivo de la autoeficacia académica. Sin embargo, se observó en la extraversión y responsabilidad valores de los coeficientes beta que mostraron débil valor predictivo (.19 y .12, respectivamente). En consecuencia, se decidió eliminar del modelo 3, estos dos tipos de personalidad y solo se integró la apertura. Los resultados mostraron que dentro del modelo 3 , las variables actividades $(\beta=.60, \mathrm{t}=11.48, \mathrm{p}<.000)$, afecto positivo $(\beta=.26, \mathrm{t}=9.85, \mathrm{p}<.000)$ y apertura $(\beta=.17, \mathrm{t}=6.99, \mathrm{p}<.000)$. Finalmente, el tamaño del efecto de este modelo fue alto $\left(f^{2}=.43\right)$. Lo que determina que un modelo integrado por la autorregulación de actividades, afecto positivo y un tipo de personalidad de apertura explican el $29 \%$ de la varianza de la autoeficacia académica. 
Tabla 3

Resultados de regresión jerárquica usando la autoeficacia académica como criterio

\begin{tabular}{|c|c|c|c|c|c|c|c|}
\hline Predictor & B & $\beta$ & $\mathrm{t}$ & $\bar{r}$ & $R^{2}$ [IC 95\%] & $\Delta R^{2}$ & $f^{2}$ \\
\hline $\begin{array}{l}\text { Modelo } 1 \\
\text { (Constante) }\end{array}$ & $22.00^{* *}$ & & $21.53^{* *}$ & & & & \\
\hline Autorregulación de act. & $0.50^{* *}$ & 0.43 & $16.84^{* *}$ & $.43^{* *}$ & $.19[.15, .23]$ & - & .24 \\
\hline $\begin{array}{l}\text { Modelo } 2 \\
\text { (Constante) }\end{array}$ & $16.32^{* *}$ & & $14.82^{* *}$ & & & & \\
\hline Autorregulación de act. & $0.37^{* *}$ & 0.32 & $12.26^{* *}$ & $.43^{* *}$ & & & \\
\hline Afecto positivo & $0.43^{* *}$ & 0.29 & $11.04^{* *}$ & $.41^{* *}$ & $.26[.22, .30]$ & .074 & .36 \\
\hline $\begin{array}{l}\text { Modelo } 3 \\
\text { (Constante) }\end{array}$ & $12.62^{* *}$ & & $10.49^{* *}$ & & & & \\
\hline Autorregulación de act. & $0.34^{* *}$ & 0.60 & $11.48^{* *}$ & $.43^{* *}$ & & & \\
\hline Afecto positivo & $0.38^{* *}$ & 0.26 & $9.85^{* *}$ & $.41^{* *}$ & & & \\
\hline Apertura & $0.40^{* *}$ & 0.17 & $6.99^{* *}$ & $.30^{* *}$ & $.29[.25, .33]$ & .028 & .43 \\
\hline
\end{tabular}

\section{Discusión}

Inicialmente, el estudio pretendió demostrar el planteamiento de un modelo explicativo de la autoeficacia académica. Se propuso estudiar el proceso conductual basándose de la literatura, lo que permitió conocer que la autorregulación de actividades se compone de indicadores de acciones académicas (Dominguez-Lara et al., 2014). De esa manera, se planteó el primer modelo del estudio, el cual determinó que la respuesta conductual (autorregulación de actividades) explicaba el 19\% de la varianza de la autoeficacia académica. Lo que concuerda con Zimmerman et al. (2005) en que la planificación y organización del tiempo y estudio forma parte de la autoeficacia del estudiante. Además, estudios han mostrado que la relación entre la autoeficacia y autorregulación académica (Alegre, 2014; Fernández \& Bernardo, 2011) es igual de estable que en este estudio ( $r=.43)$.

El segundo modelo planteado integra el afecto positivo como el procesamiento emocional junto al conductual del modelo 1. La revisión de la literatura ha mostrado que la autoeficacia se relaciona con el afecto (Caprara et al., 2006). Inclusive, el afecto positivo llegó a ser una variable mediadora de la autoeficacia y conducta (He et al., 2020); así mismo, proporciona beneficios en la salud física y mental (Krok \& Zarzycka, 2020). Dentro del modelo 2, se incrementó en 7.4\% el valor predictivo del modelo 1 a la varianza en la autoeficacia académica. Por lo cual, se ha comprobado que el afecto positivo cumple un rol importante como predictor. Los resultados muestran congruencia con otro estudio que mostró el efecto de las emociones positivas en la autoeficacia; además, de su vínculo con el compromiso académico, lo cual genera un adecuado desenvolvimiento estudiantil (Oriol-Granado, Mendoza-Lira, Covarrubias-Apablaza, \& MolinaLópez, 2017). Dichos resultados aportan a las teorías sobre el proceso de aprendizaje a través de la autoeficacia académica (Bower, 1981); en otras palabras, la autoeficacia integra el componente emocional el cual influye en el proceso de aprendizaje (Bandura, 1997).

En relación con el modelo 3, se añadió la variable de tipos de personalidad (extraversión, responsabilidad y apertura). A su vez, los resultados correlacionales mostraron la relación moderada de la autoeficacia académica con la personalidad, especialmente con extraversión, responsabilidad y apertura, lo que corrobora los resultados de Zakiei et al. (2020) y Francescato et al. (2020). No obstante, el modelo 3 inicial mostró que la responsabilidad y extraversión eran variables con baja carga predictiva; por ello se decidió extraerlos del modelo. Lo cual discrepa 
con los resultados de Francescato et al. (2020) quien menciona que la responsabilidad y extraversión son predictores de la autoeficacia. Estos resultados se deben a que el autor ha evaluado la autoeficacia en una situación diferente a la académica; en una situación educativa son diferentes factores de la personalidad que pueden influir en la autoeficacia del estudiante. Por otro lado, se consideró conveniente no incluir del modelo a la amabilidad y el neuroticismo, debido a que la primera posee correlación débil y casi nula, y la segunda posee correlación negativa (Judge \& Ilies, 2002; Zakiei et al., 2020). Al realizar esos cambios, el modelo 3 explicó el 29\% de la varianza de la autoeficacia académica, siendo el máximo valor predictivo del $33 \%$ con un índice de confianza del $95 \%$. Este resultado puede deberse a que la apertura como tipo de personalidad involucra procesos del intelecto e imaginación (Martínez-Molina \& Arias, 2018), los cuales pueden influir en sus creencias de autoeficacia y desarrollo académico, a diferencia de la responsabilidad y extraversión. No obstante, ha sido conveniente dejar abierta esta inferencia para el interés de otros estudios.

\section{Conclusión e implicancia académica}

En primer lugar, los resultados permiten observar que el proceso cognitivo, emocional y conductual son necesarios para lograr que el estudiante se auto perciba de forma positiva y de esa manera pueda desenvolverse mejor en su vida académica. Se debe considerar que la autorregulación de actividades académicas es un proceso de orden y disciplina que el estudiante utiliza para obtener logros académicos personales. De igual modo, el afecto positivo conlleva un proceso emocional que marca la tendencia actitudinal y motivacional del estudiante, la cual se ve reflejado en su autoeficacia a la hora de enfrentar problemas académicos. A su vez, la extraversión, responsabilidad y apertura permiten conocer que la personalidad forma parte fundamental del estudiante para lograr el éxito académico. Sin embargo, entre todas las variables tuvo un menor poder predictivo en la autoeficacia.

En segunda instancia, estos resultados deben incitar a las instituciones y docentes a considerar que el estudiante motivado y con la capacidad de creer en sí mismo puede mejorar su desempeño académico. De igual forma, se debe tomar en cuenta que el recurso cognitivo y conductual del estudiante se activa por medio del afecto positivo. En definitiva, no solo se deben tomar en cuenta estos tres factores explicativos de la autoeficacia académica, se tendría que considerar otras variables que han sido reportadas por la ciencia como importantes para el desarrollo de la autoeficacia y el logro académico.

\section{Limitaciones del estudio}

Existieron ciertas limitaciones que pueden ser consideradas en estudios posteriores. Por una parte, la evaluación de la autorregulación de actividades podría ser medido por otros instrumentos que tengan evidencia científica en el contexto peruano. Hasta el momento no existe una escala abreviada sobre esta variable y podría ser beneficioso para la ciencia poder tener acceso a un instrumento breve que lo mida. Por otro lado, una cantidad considerable de datos fueron excluidos por su baja calidad en las respuestas, lo que pudo evitarse con mayor control a la hora de aplicar los instrumentos.

\section{Referencias}

Alegre, A. A. (2014). Academic self-efficacy, self-regulated learning and academic performance in first-year university students. Propósitos y Representaciones, 2(1), 101-120. https://doi.org/10.20511/pyr2014.v2n1.54

Alvarado-Ochoa, W., González-Gordillo, W., Fontaines-Ruiz, T., Salamea-Nieto, R., \& BlacioAguilar, G. (2018). Personalidad del estudiante exitoso. Revista Ciencia UNEMI, 10(25). https://doi.org/10.29076/issn.2528-7737vol10iss25.2017pp89-96p

Bandura, A. (1986). Social foundations of thought and action: A social cognitive theory. PrenticeHall Inc. https://psycnet.apa.org/record/1985-98423-000 
Bandura, A. (1997). Self-efficacy: The exercise of control (1st ed.). W. H. Freeman and Company. Bandura, A. (2006). Guide for constructing self-efficacy scales. In T. Urdan \& F. Pajares (Eds.), Self-efficacy beliefs of adolescents (pp. 307-337). Information Age Publishing.

Bonetto, V. A., Paoloni, P. V., \& Donolo, D. S. (2017). Creencias de autoeficacia y contextos de evaluación. Un estudio con alumnos universitarios. Actualidades Investigativas En Educación, 17(2). https://doi.org/10.15517/aie.v17i1.28144

Bower, G. H. (1981). Mood and memory. American Psychologist, 36(2), 129-148. https://doi.org/10.1037/0003-066X.36.2.129

Brígido, M., \& Borrachero, A. B. (2011). Relación entre autoconcepto, autoeficacia y autorrgulación en ciencias de futuros maestros de primaria. International Journal of Developmental and Educational Psychology, l(2), 107-113. https://dialnet.unirioja.es/servlet/articulo?codigo=5988648

Caprara, G. V., Barbaranelli, C., Pastorelli, C., \& Cervone, D. (2004). The contribution of selfefficacy beliefs to psychosocial outcomes in adolescence: predicting beyond global dispositional tendencies. Personality and Individual Differences, 37(4), 751-763. https://doi.org/10.1016/j.paid.2003.11.003

Caprara, G. V., Steca, P., Gerbino, M., Paciello, M., \& Vecchio, G. M. (2006). Looking for adolescents' well-being: self-efficacy beliefs as determinants of positive thinking and happiness. Epidemiologia e Psichiatria Sociale, 15(1), 30-43. https://doi.org/10.1017/S1121189X00002013

Caprara, G. V., Vecchione, M., Alessandri, G., Gerbino, M., \& Barbaranelli, C. (2011). The contribution of personality traits and self-efficacy beliefs to academic achievement: A longitudinal study. British Journal of Educational Psychology, 81(1), 78-96. https://doi.org/10.1348/2044-8279.002004

Cassaretto, M., \& Martínez-Uribe, P. (2017). Validación de las escalas de bienestar, de florecimiento y afectividad. Pensamiento Psicológico, 15(1), 19-31. https://revistas.javerianacali.edu.co/index.php/pensamientopsicologico/article/view/125 5

Cohen, J. (1988). Statistical Power Analysis for the Behavioral Sciences. New York: Lawrence Erlbaum Associates.

Covarrubias-Apablaza, C. G., Acosta-Antognoni, H., \& Mendoza-Lira, M. (2019). Selfregulation learning and general self-efficacy and their relation with academic goals in university students. Formacion Universitaria, 12(6), 103-114. https://doi.org/10.4067/S0718-50062019000600103

Criollo, M., Romero, M., \& Fontaines-Ruiz, T. (2017). Autoeficacia para el aprendizaje de la investigación en estudiantes universitarios. Psicologia Educativa, 23(1), 63-72. https://doi.org/10.1016/j.pse.2016.09.002

Cupani, M., \& Zalazar-Jaime, M. F. (2014). Rasgos complejos y rendimiento académico: contribución de los rasgos de personalidad, creencias de autoeficacia e intereses TT. Revista Colombiana de Psicología, 23(1), 57-71. http://www.revistas.unal.edu.co/index.php/psicologia/article/view/39774/45636

Diener, E., Wirtz, D., Tov, W., Kim-Prieto, C., Choi, D., Oishi, S., \& Biswas-Diener, R. (2010). New well-being measures: Short scales to assess flourishing and positive and negative feelings. Social Indicators Research, 97(2), 143-156. https://doi.org/10.1007/s11205009-9493-y

Dominguez-Lara, S. A., Villegas, G., \& Centeno, S. B. (2014). Procrastinación académica: Validación de una escala en una muestra de estudiantes de una universidad privada. Liberabit. Revista de Psicología, 20(2), 293-304. http://www.scielo.org.pe/pdf/liber/v20n2/a10v20n2

Dominguez-Lara, Sergio Alexis, Villegas, G., Yauri, C., Mattos, E., \& Ramírez, F. (2012). Propiedades psicométricas de una escala de autoeficacia para situaciones académicas en estudiantes universitarios peruanos. Revista de Psicología, 2(1), 28-40. http://revistas.ucsp.edu.pe/index.php/psicologia/article/view/8

Dominguez, S. A. (2014). Autoeficacia para situaciones académicas en estudiantes universitarios peruanos: un enfoque de ecuaciones estructurales. Revista de Psicología, 4(4), 45-53. 
https://revistas.ucsp.edu.pe/index.php/psicologia/article/view/20

Donnellan, M. B., Oswald, F. L., Baird, B. M., \& Lucas, R. E. (2006). The Mini-IPIP scales: Tiny-yet-effective measures of the Big Five factors of personality. Psychological Assessment, 18(2), 192-203. https://doi.org/10.1037/1040-3590.18.2.192

Eich, E. (1995). Searching for Mood Dependent Memory. Psychological Science, 6(2), 67-75. http://www.jstor.org/stable/40062990

Fernández, E., \& Bernardo, A. (2011). Autoeficacia en la autorregulación del aprendizaje de estudiantes universitarios. International Journal of Developmental and Educational Psychology, 3(1), 201-208. https://www.redalyc.org/pdf/3498/349832330020.pdf

Fodor, O. C., \& Pintea, S. (2017). The "Emotional side" of entrepreneurship: A meta-analysis of the relation between positive and negative affect and entrepreneurial performance. Frontiers in Psychology, 8, 1-16. https://doi.org/10.3389/fpsyg.2017.00310

Fosse, T., Buch, R., Säfvenbom, R., \& Martinussen, M. (2016). The impact of personality and self-efficacy on academic and military performance: The mediating role of self-efficacy. Journal of Military Studies, 6(1), 47-65. https://doi.org/10.1515/jms-2016-0197

Francescato, D., Lauriola, M., Giacomantonio, M., \& Mebane, M. E. (2020). Do personality traits and personal values predict career efficacy and career progression of successful political women? An exploratory study. Personality and Individual Differences, 160. https://doi.org/10.1016/j.paid.2020.109918

Galindo, J. (2004). Apuntes de historio de una comunicología posible. Hipótesis de configuración y trayectoria. Redes.Com: Revista de Estudios Para El Desarrollo Social de La Comunicación, 12(1), https://revistasum.umanizales.edu.co/ojs/index.php/escribania/article/view/3004

George, D., \& Mallery, P. (2020). IBM SPSS Statistics 26: Step by Step. A Simple Guide and Reference (6th ed.). Taylor \& Francis.

Goldberg, L. R. (1992). The development of markers for the Big-Five factor structure. Psychological Assessment, 4(1), 26-42. https://doi.org/10.1037/1040-3590.4.1.26

He, P., Zhou, Q., Zhao, H., Jiang, C., \& Wu, Y. J. (2020). Compulsory Citizenship Behavior and Employee Creativity: Creative Self-Efficacy as a Mediator and Negative Affect as a Moderator. Frontiers in Psychology, 11. https://doi.org/10.3389/fpsyg.2020.01640

Judge, T. A., \& Ilies, R. (2002). Relationship of personality to performance motivation: A metaanalytic review. Journal of Applied Psychology, 87(4), 797-807. https://doi.org/10.1037/0021-9010.87.4.797

Kerlinger, F. N., \& Lee, H. B. (2002). Investigación del comportamiento: Métodos de investigación en ciencias sociales. McGraw-Hill.

Kline, R. B. (2016). Principles and Practice of Structural Equation Modeling (4th ed.). The Guilford Press.

Krok, D., \& Zarzycka, B. (2020). Self-Efficacy and Psychological Well-Being in Cardiac Patients: Moderated Mediation by Affect and Meaning-Making. The Journal of Psychology, 154(6), 411-425. https://doi.org/10.1080/00223980.2020.1772702

Kurtovic, A., Vrdoljak, G., \& Idzanovic, A. (2019). Predicting procrastination: The role of academic achievement, self-efficacy and perfectionism. International Journal of Educational Psychology, 8(1), 1-26. https://doi.org/10.17583/ijep.2019.2993

Lee, J. yeon, \& Ciftci, A. (2014). Asian international students' socio-cultural adaptation: Influence of multicultural personality, assertiveness, academic self-efficacy, and social support. International Journal of Intercultural Relations, 38(1), 97-105. https://doi.org/10.1016/j.ijintrel.2013.08.009

Lin, S. H., Lu, W. C., Chen, M. Y., \& Chen, L. H. (2014). Association Between Proactive Personality and Academic Self-Efficacy. Current Psychology, 33(4), 600-609. https://doi.org/10.1007/s12144-014-9231-8

Martínez-Molina, A., \& Arias, V. B. (2018). Balanced and positively worded personality shortforms: Mini-IPIP validity and cross-cultural invariance. PeerJ, 6(9), e5542. https://doi.org/10.7717/peerj.5542

Moraine, P. (2014). Las funciones ejecutivas del estudiante. Mejorar la atención, la memoria, la organización y otras funcciones para facilitar el aprendizaje. Narcea, S.A. 
Narváez, G., Posso, M., Guzmán, F., \& Valencia, S. (2018). Autoeficacia académica: un factor determinante para el ajuste académico en la vida universitaria. Revista SATHIRI: Sembrador, 13(2). https://doi.org/10.32645/13906925.755

Nawas, T., Khattak, K., \& Rehman, K. (2019). Autoeficacia empresarial como mediador en la relación de la personalidad proactiva y la intención empresarial. Evidencia de titulados universitarios. Revista Dilemas Contemporáneos: Educación, Política y Valores, 6(125), $1-23$.

http://www.dilemascontemporaneoseducacionpoliticayvalores.com/index.php/dilemas/a rticle/view/1383

Oriol-Granado, X., Mendoza-Lira, M., Covarrubias-Apablaza, C.-G., \& Molina-López, V.-M. (2017). Emociones positivas, apoyo a la autonomía y rendimiento de estudiantes universitarios: el papel mediador del compromiso académico y la autoeficacia. Revista de Psicodidáctica, 22(1), 45-53. https://doi.org/10.1016/S1136-1034(17)30043-6

Ornelas, M., Blanco, H., Gastélum, G., \& Chávez, A. (2012). Autoeficacia Percibida en la conducta Académica de Estudiantes Universitarias. Formación Universitaria, 5(2), $17-$ 26. https://doi.org/10.4067/S0718-50062012000200003

Ornelas, M., Blanco, H., Rodríguez, J. M., \& Flores, F. J. (2011). Análisis Psicométrico de la Escala Autoeficacia en Conductas de Cuidado de la Salud Física en Universitarios de Primer Ingreso. Formación Universitaria, 4(6), 21-34. https://doi.org/10.4067/S071850062011000600004

Özdemir, M., Özdemir, N., Gören, S. Ç., Ötken, Ş., Ernas, S., \& Yalçın, M. T. (2019). Relationship between Personality Traits and Administrative Self-Efficacy Beliefs among Principals. $\quad T e D$ Eğitim Ve Bilim, 45(201), 273-291. https://doi.org/10.15390/eb.2019.7864

Palenzuela, D. L. (1983). Construcción y validación de una escala de autoeficacia percibida específica de situaciones académicas. Análisis y Modificación de Conducta, 9(21), 185219. https://doi.org/10.33776/amc.v9i21.1649

Pease, M. A. (2015). El potencial que emerge: cognición, neurociencia y aprendizaje en adolescentes universitarios. In M. A. Pease, F. Figallo, \& L. Ysla (Eds.), Cognición, neurociencia y aprendizaje. El adolescente en la educación superior. Lima: Editorial Pontificia Universidad Católica del Perú.

Pecina, R. M. (2014). Auto eficacia en el aprendizaje de competencias disciplinares en estudiantes de licenciatura en enfermería en una universidad pública. Revista Iberoamericana de Producción Académica y Gestión Educativa, (1). http://www.pag.org.mx/index.php/PAG/article/view/30

Poropat, A. E. (2009). A Meta-Analysis of the Five-Factor Model of Personality and Academic Performance. Psychological Bulletin, 135(2), 322-338. https://doi.org/10.1037/a0014996

Reina, M. D. C. (2017). Percepciones de autoevaluación: Autoestima, autoeficacia y satisfacción vital en la adolescencia. Psychology, Society, \& Education, 2(1), 55. https://doi.org/10.25115/psye.v2i1.435

Rottenberg, J., \& Johnson, S. L. (2007). Emotion and psychopathology: Bridging affective and clinical science. Washington: American Psychological Association. https://doi.org/10.1037/11562-000

Rottinghaus, P. J., Jenkins, N., \& Jantzer, A. M. (2009). Relation of Depression and Affectivity to Career Decision Status and Self-Efficacy in College Students. Journal of Career Assessment, 17(3), 271-285. https://doi.org/10.1177/1069072708330463

Rottinghaus, P. J., Lindley, L. D., Green, M. A., \& Borgen, F. H. (2002). Educational aspirations: The contribution of personality, self-efficacy, and interests. Journal of Vocational Behavior, 61(1), 1-19. https://doi.org/10.1006/jvbe.2001.1843

Ruiz-Dodobara, F. G. (2005). Relación entre la motivación de logro académico, la autoeficacia y la disposición para la realización de una tesis. Persona, (8), 145-170. https://doi.org/10.26439/persona2005.n008.898

Schunk, D. H. (1991). Self-Efficacy and Academic Motivation. Educational Psychologist, 26(3), 207-231. https://doi.org/10.1080/00461520.1991.9653133

Schutte, N. S. (2013). The broaden and build process: Positive affect, ratio of positive to negative 
affect and general self-efficacy. The Journal of Positive Psychology, 9(1), 66-74. https://doi.org/10.1080/17439760.2013.841280

Shepherd, D. A., \& Patzelt, H. (2018). Entrepreneurial Cognition. Springer International Publishing. https://doi.org/10.1007/978-3-319-71782-1

Sheu, H. Bin, Liu, Y., \& Li, Y. (2017). Well-Being of College Students in China: Testing a Modified Social Cognitive Model. Journal of Career Assessment, 25(1), 144-158. https://doi.org/10.1177/1069072716658240

Sidratulmunthah, Hussain, S., \& Imran-Malik, M. (2018). Towards nurturing the entrepreneurial intentions of neglected female business students of Pakistan through proactive personality, self-efficacy and university support factors. Asia Pacific Journal of Innovation and Entrepreneurship, 12(3), 363-378. https://doi.org/10.1108/apjie-032018-0015

Ümmet, D. (2017). Structural relationships among counselling self-efficacy, general self-efficacy and positive-negative affect in psychological counsellor candidates. Kuram ve Uygulamada Egitim Bilimleri, 17(6), 1875-1892. https://doi.org/10.12738/estp.2017.6.0180

Valdivieso, J. A., Carbonero, M. A., \& Martín-Antón, L. J. (2013). Elementary School Teachers' Self-Perceived Instructional Competence: A New Questionnaire. Revista de Psicodidáctica, 18(1), 47-78. https://ojs.ehu.eus/index.php/psicodidactica/article/view/5622/6212

Zakiei, A., Vafapoor, H., Alikhani, M., Farnia, V., \& Radmehr, F. (2020). The relationship between family function and personality traits with general self-efficacy (parallel samples studies). BMC Psychology, 8(88), 1-11. https://doi.org/10.1186/s40359-020-00462-w

Zarza-Alzugaray, F. J., Bustamante, J. C., Casanova, O., \& Orejudo, S. (2018). La autoeficacia creativa y la autoestima como predictores de la capacidad creativa en estudiantes universitarios. REIDOCREA, 8, 7-14. https://www.ugr.es/ reidocrea/8-2.pdf

Zhang, R. P. (2016). Positive Affect and Self-Efficacy as Mediators Between Personality and Life Satisfaction in Chinese College Freshmen. Journal of Happiness Studies, 17(5), 20072021. https://doi.org/10.1007/s10902-015-9682-0

Zimmerman, B. J., \& Kitsantas, A. (2005). Homework practices and academic achievement: The mediating role of self-efficacy and perceived responsibility beliefs. Contemporary Educational Psychology, 30(4), 397-417. https://doi.org/10.1016/j.cedpsych.2005.05.003

Zimmerman, B. J., Kitsantas, A., \& Campillo, M. (2005). Evaluación de la Autoeficacia Regulatoria: Una Perspectiva Social Cognitiva. Revista Evaluar, 5(1). https://doi.org/10.35670/1667-4545.v5.n1.537

Zuffianò, A., Alessandri, G., Gerbino, M., Luengo-Kanacri, B. P., Di Giunta, L., Milioni, M., \& Caprara, G. V. (2013). Academic achievement: The unique contribution of self-efficacy beliefs in self-regulated learning beyond intelligence, personality traits, and self-esteem. Learning and Individual Differences, 23(1), 158-162. https://doi.org/10.1016/j.lindif.2012.07.010 